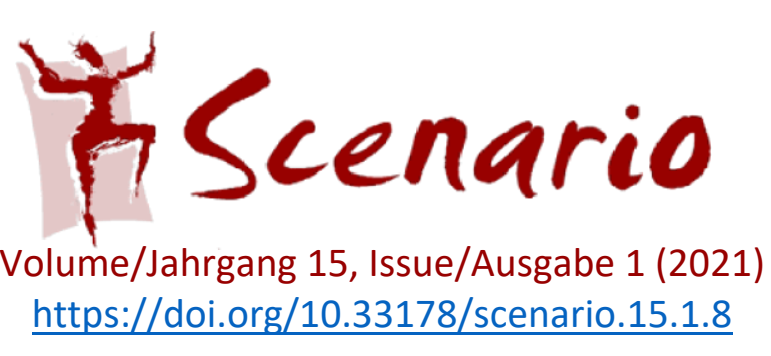

\title{
Rezension: Hensel, A. (2020). Fremdsprachen- unterricht als Ereignis. Zur Fundierung einer performativ-ästhetischen Praxis. Schibri-Verlag.
}

\author{
Leticia García Brea, Stefanie Giebert
}

Die in Buchrezensionen vertretenen Ansichten und Meinungen sind die der jeweiligen Rezensent:innen und reflektieren nicht notwendigerweise die Position von SCENARIO.

„Wie und inwiefern begünstigt meine an der Universität Göttingen entwickelte performativästhetische Unterrichtspraxis die Förderung von effektiven Sprachlernprozessen und auch eine Persönlichkeitsentwicklung bei den Lernenden? Inwiefern lässt sie sich als innovativer Beitrag zu einer performativen Lehr und Lernkultur begreifen?" (Hensel, 2020, S. 17) Das sind die Fragen, die Alexandra Hensel veranlasst haben, ihr Forschungsprojekt durchzuführen. Die Forschungsarbeit der Autorin, die an der Abteilung für Interkulturelle Germanistik, Lektorat Deutsch als Fremdsprache, der Georg-August-Universität Göttingen entwickelt wurde, wurde in der Scenario-Buchreihe veröffentlicht, die laut den Herausgeber:innen der Serie "die weitere Fundierung der Theorie und Praxis performativen Lehrens, Lernens und Forschens vorantreiben soll“ (Hensel, 2020, S. 9). Das Buch bezieht sich auf ihre eigene Unterrichtspraxis, den von ihr konzipierten Deutschkurs Deutsch lernen durch Theaterspiel für internationale Studierende und Universitätsmitarbeiter:innen auf B2-/C1-Niveau. Der Kurs wurde von der Autorin in einer Zeitspanne von acht Jahren insgesamt vierzehn Mal vom Sommersemester 2008 bis zum Sommersemester 2016, angeboten. Das in der Dissertation untersuchte Material stammt aus den letzten vier Semestern des Kurses.

Was bedeutet es, den Unterricht, wie die Autorin darstellt, als „Ereignis“ zu sehen? Hensel fand in den Worten von Theaterwissenschaftlerin Erika Fischer-Lichte die Grundidee für die Entwicklung ihres Projektes: Theateraufführungen sind ein komplexes Ereignis „von den AkteurInnen und ZuschauerInnen gemeinsam hervorgebracht, wobei mehrere Komponenten wie Körper, Stimme, Raum und Licht zusammenspielen“ (Fischer-Lichte, 2004, S. 47, zitiert nach Hensel, 2020, S. 12). Dieses Zusammenspiel kann ein gemeinsames besonderes Erlebnis für alle Beteiligten ermöglichen, das laut Hensel „so nur in dieser Form, in diesem Moment stattfinden kann“ (S. 13). Auf dieser Grundidee basiert das Konzept für den Kurs Deutsch lernen durch Theaterspiel, indem Inhalte „nicht nur vermittelt, sondern gemeinsam vom Ensemble der Lehrenden und Lernenden hervorgebracht“ (S. 13) werden. 
Im ersten von neun Kapiteln werden der Rahmen der Forschungsarbeit und der Untersuchungsgegenstand ausführlich beschrieben. In Kapitel 2 präsentiert die Autorin die theoretischen Grundlagen, auf denen ihre Forschung und Vorgehensweise basieren. Hier beschreibt sie u.a. einen interdisziplinären Bezugsrahmen, der ihre Lehrpraxis und Forschungsarbeit „in der aktuellen Forschungsdiskussion theoretisch verankert, wobei Neurowissenschaften, Fremdsprachendidaktik, Pädagogik, Theaterwissenschaften sowie Theaterpädagogik zu Bezugsdisziplinen werden“ (S. 18). Dieses Kapitel ist eine gute Einführung in den theoretischen Hintergrund der Arbeit und zusammen mit dem sehr ausführlichen Literaturverzeichnis bietet es einen hilfreichen Überblick in Bezug auf die Fachdiskussion zur Rolle von Drama und Theater in der Sprach-, Literatur- und Kulturvermittlung.

Im Kapitel 3 begründet die Autorin das von ihr gewählte Forschungsdesign. Es wurden drei Perspektiven trianguliert, um Daten zu Sprechbereitschaft, Sprachbewusstsein, Sprechkompetenz, Persönlichkeitsentwicklung und die Wirkung einer performativästhetischen Unterrichtspraxis zu generieren: Beobachtungen der Lehrkraft, Videoaufnahmen sowie das Feedback der Teilnehmenden.

Im Mittelpunkt des nächsten Kapitels steht die ausführliche Beschreibung des Kursverlaufes mit allen Phasen, von denen Lehrkräfte, die einen ähnlichen Kurs planen möchten, ganz sicher profitieren können. Dabei beschreibt die Autorin die erste Sitzung, die dem Kennenlernen von Teilnehmenden und Kursinhalten dient, im Detail geschildert, mit einer genauen Auflistung aller Übungen und ihrer Bedeutung. In den nächsten Abschnitten geht es um die Formsuche (S. 99) und Formfindung. Hier beschreibt sie beispielhaft, wie eine Unterrichtsstunde abläuft (Aufwärmen, Textarbeit und Reflexion). Auch schildert sie, welche Texte die Studierenden zur szenischen Interpretation durch improvisiertes Spiel (dies ist der Hauptfokus der Sitzungen) erhalten und wie die Kursleiterin daraus eine ca. 20-minütige Werkschau zusammenstellt. Auch die Werkschau, die Präsentation der gemeinsam erarbeiteten Szenen, wird anhand eines konkreten Beispiels aus einem Semester erläutert. Die Bedeutung dieses Endproduktes für die Motivation der Teilnehmenden hebt die Autorin hervor. Alexandra Hensel stellt hier dar, worin sie die Potentiale ihres Kurses für die Teilnehmenden sieht. Außerdem umreißt sie die ihrer Meinung nach bestehenden Anforderungen an eine Lehrkraft, die Unterricht performativ gestalten möchte. Dieser Abschnitt könnte für Lehrpersonen, die performative Techniken ausprobieren möchten, hilfreich sein, da sie die von der Autorin genannten Anforderungen mit ihrer eigenen Lehrphilosophie vergleichen und dann vielleicht leichter entscheiden können, ob performative Methoden für sie ,passen'.

In Kapitel 5 berichtet die Autorin, welche Erkenntnisse sie aus videografierten Unterrichtsausschnitten ziehen konnte, unter anderem die durch die Längsschnittperspektive (mehrere Aufnahmen im Verlauf eines Semesters) erkennbare Entwicklung der 
Teilnehmenden in sprachlicher und persönlicher Hinsicht. So wurden zum Beispiel die Texte lauter vorgetragen, sprachliche Fehler eliminiert, Charaktere und humorvolle Passagen von den spielenden Studierenden deutlicher ausgearbeitet. Was die Autorin nicht direkt beschreibt, aber man aufgrund der anderen Kapitel vermuten kann, ist, dass dies durch wiederholte Feedbackrunden zur Szene mit der gesamten Gruppe über mehrere Unterrichtsstunden und privates Proben sowie Reflexionsfragen der Lehrkraft (z.B. „was könnte man noch deutlicher machen“) zustande gekommen ist.

Die dritte Säule des Forschungsdesigns bildet in Kapitel 6 die Perspektive der Teilnehmenden auf den Kurs, die durch Fragebögen mit offenen Fragen (von drei aus vierzehn Semestern) sowie vier Reflexionsschreiben von Studierenden abgedeckt wird. Hier schildert Alexandra Hensel, dass zu Anfang des Kurses Gefühle wie Angst und Stress beim Sprechen der Fremdsprache vorherrschen und die Teilnehmenden am Ende mehrheitlich von sprachlicher Verbesserung, weniger Angst und mehr positiven Emotionen beim Benutzen der Fremdsprache (im Kurs) berichten.

Bei der zweiten und dritten Säule des Forschungsdesigns scheinen einige Schwächen zu bestehen, die auch teilweise von der Autorin selbst angemerkt werden, so etwa, dass das videografierte Material sehr kurz ist und dass an den Fragebögen zwischen den Erhebungen noch Änderungen vorgenommen wurden.

Zum Beispiel dokumentiert die Videografie, wie die sprachliche Umsetzung einer von zwei Studierenden frei improvisierten Szene zwischen der ersten Probe und der Generalprobe und Aufführung sicherer und komplexer wird. Dies als Beispiel für eine individuelle Entwicklung zweier Studierender zu interpretieren ist sicher legitim, aber um Videografie sinnvoll als Datenerhebungsinstrument einzusetzen, hätte viel mehr Material aufgenommen und ausgewertet werden müssen. Dies merkt die Autorin in Kapitel 7 auch selbstkritisch an, begründet es aber mit einem Mangel an Ressourcen. Vielleicht hätte sie in diesem Fall auf die Videografie lieber ganz verzichten sollen?

Andere Aspekte, die in der Methodenreflektion kurz erwähnt werden, sind beispielsweise, dass die Instrumente nur punktuell eingesetzt wurden („in einem kleinen Rahmen“, S. 221): die Fragebögen in drei von vierzehn Semestern und die Videografie in einem von vierzehn Semestern.

Teilweise werden in den Kapiteln 5 und 6 Schlussfolgerungen gezogen, die die Autorin in ihrer Reflexion in Kapitel 7 nicht hinterfragt, beispielsweise, dass 44 Fragebögen vom Anfang der Semester vorliegen, aber nur noch 23 vom zweiten Befragungszeitpunkt (Semesterende). Es scheint daher etwas problematisch, hier Aussagen zu treffen wie: „Insgesamt lassen sich bei 91\% konkrete Veränderungen bezüglich eines besseren Gefühls ausmachen“ (S. 208) - es sind 
vermutlich 91\% der 23 verbliebenen Studierenden, aber was ist mit den Studierenden, die an der zweiten Umfrage nicht teilgenommen haben? Diese Studierenden haben den Kurs aus verschiedenen der Autorin bekannten (z.B. Verpflichtungen in anderen Lehrveranstaltungen) oder unbekannten Gründen abgebrochen. Möglich ist allerdings, dass diese Studierenden den Kurs weniger positiv beurteilt hätten.

In den oben genannten Punkten zeigt sich insgesamt eine Problemlage, die vermutlich typisch für Aktionsforschungsprojekte in der Dramapädagogik ist: Forschende Lehrkräfte haben häufig einfach nicht die Ressourcen, um methodisch einwandfreie empirische Forschungsprojekte durchzuführen, da die Erhebung im eigenen Unterricht stattfindet und weder Extra-Personal noch Geld noch Zeit für z.B. längere Videobegleitung oder komplexere Befragungen zur Verfügung stehen.

In Kapitel 7 fasst die Autorin nach der Reflexion ihrer Methoden als Nächstes das Wechselspiel zwischen performativ-ästhetischer Unterrichtspraxis und Fremdsprachenunterricht noch einmal zusammen. Die Autorin resümiert, dass die Wirkung der Theaterarbeit „intensiv sein [kann], doch ist sie von Individuum zu Individuum verschieden“ (S. 231). Es scheint, dass die Autorin hier sagen möchte: Es können bei manchen Lernenden intensive/starke Veränderungen in Sprechbereitschaft, Sprachbewusstsein, Sprechkompetenz und Persönlichkeitsentwicklung auftreten, aber nicht bei allen Teilnehmern werden alle Bereiche verändert und nicht im gleichen Maße.

Kapitel 8 greift noch einmal die wichtigsten Erkenntnisse heraus und besonders interessant erscheint hier - vor allem für noch unerfahrene Lehrkräfte - dass die performative Unterrichtsform als ,in regulären Räumen und Zeitfenstern durchführbar“ (S. 233) angesehen wird. Dies bedeutet also, dass eine Kursaufführung nicht notwendigerweise in einem Theaterraum stattfinden muss, denn Cafeterien oder sogar Korridore eignen sich ebenfalls, oder dass außercurricular Extrazeit für das Aufführungsprojekt eingeplant werden müsste. Es kann innerhalb eines Kurses mit zwei SWS (im deutschen Uni-Kontext meist: 1x pro Woche 90 Minuten) realisiert werden.

Im letzten Kapitel wird eine unterrichtspraktische Handreichung angeboten, die eine Orientierungshilfe sein soll, „um die Bedeutung einer performativ-ästhetischen Ausrichtung von Unterricht zu bekräftigen“ (S. 234). Dieses Modell unterstützt die Idee von der Lehrkraft als performativ-ästhetische/r Übungsleiter:in und listet, neben der nötigen Grundeinstellung, die Fähigkeiten und Tätigkeiten auf, die ein Kompetenzprofil derartig konturieren sollten. Die Übungen, die alphabetisch im Kapitel 9.3 zusammengefasst sind, wurden von der Autorin mehrfach in ihrem Kurs ausprobiert, können unmittelbar und in vielen Bildungskontexten 
eingesetzt werden und sind in drei Kategorien geteilt: Übungen für das Warm-Up, Text und Szenenarbeit vorbereitende Übungen und Übungen zu Figuren- bzw. Szenenentwicklung.

Obwohl einige Punkte der Untersuchung kritisch zu sehen sind, ist das Buch, als Praxishandreichung auf jeden Fall gelungen. Es ist in erster Linie interessant für Lehrkräfte, die an Hochschulen oder in der Erwachsenenbildung tätig sind und auf Niveau B2/C1 unterrichten. Die Inhalte der letzten Kapitel in Form eines Kursverlaufes für ein Semester lassen sich aber von Fachkolleg:innen „an die curricularen Bedingungen an ihren Institutionen anpassen“ (S. 235). Das Buch ist in einem gut lesbaren Stil, ohne übermäßigen akademischen Jargon, dafür aber mit persönlicher Note geschrieben und lässt die Begeisterung der Autorin für ihr Thema deutlich erkennen. Es wird auch klar, dass sie sich der mit performativästhetischer Unterrichtsgestaltung verbundenen Herausforderungen bewusst ist, zum Beispiel in der Beschreibung des Profils einer Lehrkraft, die Unterricht performativ gestalten möchte.

Auch für Lesende, die bereits über Erfahrungen mit performativer Unterrichtsgestaltung verfügen, enthält Hensels Buch viele wertvolle Anregungen und konkrete Tipps, wie sich Fremdprachenunterricht als Ereignis inszenieren lässt.

\section{Bibliografie}

Fischer-Lichte, E. (2004). Ästhetik des Performativen. Suhrkamp. 